Original article

\title{
An 8-year study on the prevalence and drug resistance of mycobacteria in clinical specimens (2011-2018)
}

\author{
Jafar Aghajani ${ }^{\mathrm{a}, *}$, Shima Saif ${ }^{\mathrm{a}}$, Parissa Farnia ${ }^{\mathrm{a}}$, Poopak Farnia ${ }^{\mathrm{b}}$, Jalaledin Ghanavi ${ }^{\mathrm{a}}$, \\ Ali Akbar Velayati ${ }^{\mathrm{a}}$ \\ ${ }^{\mathrm{a}}$ Mycobacteriology Research Center (MRC), National Research Institute of Tuberculosis and Lung Disease (NRITLD), Shahid Beheshti University of Medical Sciences, \\ Tehran, Iran \\ ${ }^{\mathrm{b}}$ Department of Biotechnology, School of Advanced Technology in Medicine, Shahid Beheshti University of Medical Sciences, Tehran, Iran
}

\section{A R T I C L E I N F O}

\section{Keywords:}

Non-tuberculous mycobacteria

Multiplex allele specific polymerase chain reaction (MAS-PCR)

Drug resistance

INH

RIF

\begin{abstract}
A B S T R A C T
Non-tuberculous mycobacteria cause a wide range of clinical disorders. Isoniazid (INH) and rifampin (RIF) are the most effective first-line antibiotics against for Mycobacterium tuberculosis. The aim of this study was to investigate the prevalence of mycobacteria in clinical samples collected during 8 years (2011-2018) in the National Research Institute of Tuberculosis and Lung Disease, Tehran, Iran and to determine the tuberculosis drug resistance to first-line antibiotics, INH and RIF. In this study, 15829 different clinical specimens were collected at the NRITLD National Tuberculosis Center. A multiplex allele specific polymerase chain reaction (MAS-PCR) was used to identify mutations related to RIF and INH resistance. The genes involved were $k a t G 315$, inhA for INH and rpoB516, rpoB526 and rpoB531 for RIF. In total (7528/15829, 47.56\%), mycobacterial isolates including 6937 MTBC (43.82\%) and 591 NTM (3.73\%) were obtained. The frequency of MTBC isolates decreased from $65.17 \%$ (2015/3092) in 2011 to $47.06 \%$ (1224/2601) in 2018. Among NTM isolates, M. simiae was the most prevalent with 55.33\% (327/591). The average INH resistance ratio between 7528 MTB and NTM isolates was $21 \%$, from $15.98 \%$ in 2011 to $18.76 \%$ in 2018 . In the case of RIF, the same resistance trend has been gradually increasing from $12.45 \%$ in 2011 to $14.55 \%$ in 2018 . The prevalence of MDR TB has increased during the study period, from $6.49 \%(134 / 2065)$ in 2011 to $12.58 \%(174 / 1354)$ in 2018 . The results of this study indicate that early detection of mycobacterial strains and determination of their drug resistance are necessary.
\end{abstract}

\section{Introduction}

Mycobacterium species, including more than 150 species, many of them cause disease. ${ }^{1,2}$ The majority of these species do not cause disease but may, under certain conditions, become opportunistic pathogens and cause infection. ${ }^{3}$ Non-tuberculous mycobacteria (NTMs) are opportunistic pathogens that cause skin and chronic lung infections. These species are widely present in the environment. ${ }^{4}$

Because of the similar clinical pulmonary and X-ray symptoms of NTM infection with MTBC, NTM is not as prominent as TB. As a matter of fact, NTM infection is generally ignored. Significantly, there is a reciprocal trend between the prevalence of MTBC and NTM, while the rate of NTM isolates has doubled. ${ }^{4}$

Reports of non-tuberculosis mycobacterial infections worldwide have increased over the past two decades. ${ }^{5}$ This type is also isolated from clinical specimens, but cannot always be considered a disease because NTMs are capable of colonization in various parts of the body such as the respiratory tract, digestive tract and skin6,7. Treatment of patients infected with different strains of NTM is very difficult and complex because of the effective antibiotic strains that should be used. One of the reasons for the different percentages of resistance of isolated strains to different drugs is the variety of the species isolated. ${ }^{7-12}$

Patients with NTM require frequent and intensive health care resources such as hospitals and administrative visits, as well as complex treatment. These challenges also exist when associated with multiple infections, which are common in this population. The emergence of drug resistance has gradually become an obstacle to TB control, especially multidrug resistance (MDR). ${ }^{4,13-15}$

Clinically, rapid diagnosis and drug-susceptibility testing (DST) are crucial for infection control and drug treatment in Mycobacterium tuberculosis and non-tuberculosis mycobacteria. ${ }^{16}$ The increasing isolation and detection of non-tuberculous mycobacteria from patients with mycobacterial infections in Iranian laboratories reflects the fact that most patients are treated with inappropriate and unnecessary

\footnotetext{
* Corresponding author.

E-mail address: j.aghajani@theaasm.org (J. Aghajani).
} 
medication. ${ }^{15,17-21}$

Non-tuberculous mycobacteria are one of the most important causes of death, which are often a form of progressive pulmonary disease. ${ }^{22,23}$ Diagnosing NTM infection can be quite challenging. First, NTM culture can be misleading because these bacteria are present in any environment, so clinical specimens can be contaminated with unrealistic factors. Contamination may occur before, during, and even after sampling. For example, sputum samples collected may be contaminated with fresh water mouthwash. ${ }^{22}$ In order to distinguish between contamination and infection, pulmonary NTM disease diagnosis must be considered a combination of clinical, radiological, bacteriological, and histological criteria. $^{24-27}$

The use of simple, fast, and efficient tools for the differentiation of mycobacteria such as the World Health Organization (WHO), DST and line probe assay (LPA) is highly needed, especially in developing countries with high TB and Non-tuberculosis mycobacteria burden. ${ }^{28,29}$

In countries with endemic tuberculosis, isolation of non-tuberculous mycobacteria from clinical specimens, especially respiratory specimens, is not uncommon. ${ }^{20,24,29,30}$ However, in clinical conditions, in patients with suspected TB, physicians rely on local epidemiological knowledge to assess whether positive culture is related to Mycobacterium tuberculosis or NTM. Therefore, the prevalence of M. tuberculosis and NTM isolation should be studied to better understand the epidemiological features of these diseases. ${ }^{19,31}$

Non-tuberculous mycobacteria are one of the most important human pathogens in non-endemic and endemic areas of TB such as Iran. It is well known that the determination of the drug sensitivity of nontuberculosis mycobacterial clinical isolates is one of the key points in the treatment of these diseases. ${ }^{24}$ In Iran (where endemic tuberculosis is present) all patients with mycobacterial infections are treated with firstline anti-TB drugs, while most non-TB isolates have been found to be resistant to first-line anti-TB drugs(24). Therefore, accurate identification of these clinical specimens can be a great step in early diagnosis and treatment of the disease.

In this study, bacterial infection and drug resistance data are reviewed to provide an appropriate solution for the control of mycobacterial infections. It is recommended that Identification, detection and sensitivity tests for NTMs should be standardized and implemented as standard operating protocols in clinical and laboratory settings to counter the increased NTM infections. The aim of the present study was to evaluate the frequency and prevalence of clinical isolates of Mycobacteria and their drug resistance pattern from 2011 to 2018, so as to be a strategy for their control process.

\section{Method and material}

The clinical samples studied in this manuscript were collected from the National Research Institute of Tuberculosis and Lung Disease, Tehran, Iran (NRITLD) between 2011 and 2018.

Samples were collected from patients suspected of Mycobacteria and hospitalized in the hospital and clinic and transferred to the Mycobacteriology Research Center (MRC) Laboratory for bacterial culture, identification and antibiotic susceptibility testing.

A total of 15829 specimens were collected, including 12830 sputum (81.05\%), 152 pleural effusion (0.96\%), 1087 bronchial specimens (6.87\%), 169 Biopsy specimens (1.07\%) and others.

Initial isolation of Mycobacterium strains was performed by Petrov method using Leven-Stein Johnson medium. ${ }^{32,33}$ Subsequently, mycobacterial DNA was extracted from patient samples. To identify Mycobacterium species, first a $190 \mathrm{bp}$ fragment of IS6110 gene was used by primers Tb 1: 5'- ATC CTG CGA GCG TAG GCG TCGG - $3^{\prime}$ and Tb2: $5^{\prime}$ CAG GAC CAC GAT CGC TGA TCC GG $-3^{\prime} .^{33,34}$

PCR mix were used with a final volume of $50 \mu \mathrm{l}$ containing 8 picomol of Tb1 and $\mathrm{Tb} 2$ primer. $1.5 \mathrm{mM}$ magnesium chloride, 1 unit Taq polymerase enzyme (Synagen Corporation), $0.2 \mathrm{mM}$ dNTP, $1.5 \mathrm{mM}$ buffer $10 \mathrm{X}$ and $2 \%$ DMSO. The PCR reaction in Thermal Cycler (Astec
Table 1

Positive isolation rate of mycobacterial from year 2011-2018.

\begin{tabular}{lllll}
\hline \multirow{2}{*}{ year } & \multirow{2}{*}{ No. of specimen } & \multicolumn{2}{l}{ No. of isolates $(\%)^{\mathrm{a}}$} \\
\cline { 3 - 5 } & & M.TB & NTM & total \\
\hline $\mathbf{2 0 1 1}$ & 3092 & $2015(65.17)$ & $50(1.62)$ & $\mathbf{2 0 6 5}(66.79)$ \\
$\mathbf{2 0 1 2}$ & 399 & $171(42.86)$ & $10(2.51)$ & $\mathbf{1 8 1}(45.36)$ \\
$\mathbf{2 0 1 3}$ & 1131 & $526(46.51)$ & $25(2.21)$ & $\mathbf{5 5 1}(48.72)$ \\
$\mathbf{2 0 1 4}$ & 2843 & $237(8.34)$ & $31(1.09)$ & $\mathbf{2 6 8}(9.43)$ \\
$\mathbf{2 0 1 5}$ & 1120 & $511(45.63)$ & $94(8.39)$ & $\mathbf{6 0 5}(54.02)$ \\
$\mathbf{2 0 1 6}$ & 1927 & $884(45.87)$ & $114(5.92)$ & $\mathbf{9 9 8}(51.79)$ \\
$\mathbf{2 0 1 7}$ & 2716 & $1369(50.41)$ & $137(5.04)$ & $\mathbf{1 5 0 6}(55.45)$ \\
$\mathbf{2 0 1 8}$ & 2601 & $1224(47.06)$ & $130(5)$ & $\mathbf{1 3 5 4 ( 5 2 . 0 6 )}$ \\
total & $\mathbf{1 5 8 2 9}$ & $\mathbf{6 9 3 7}(43.82)$ & $\mathbf{5 9 1 ( 3 . 7 3 )}$ & $\mathbf{7 5 2 8}(47.56)$ \\
\hline
\end{tabular}

M.TB = mycobacterium tuberculosis group, NTM = nontuberculous mycobacteria.

a Numbers in the parenthesis indicaed the corresponding positive isolation rate to the number of specimens each year.

Japan) is as follows: The first cycle was $95{ }^{\circ} \mathrm{C}$ for $10 \mathrm{~min}$ to an initial denaturation followed by 35 cycles of $93^{\circ} \mathrm{C}$ for $20 \mathrm{~s}, 65^{\circ} \mathrm{C}$ for $1 \mathrm{~min}$ and $72{ }^{\circ} \mathrm{C}$ for $20 \mathrm{~s}$, and the last cycle for $5 \mathrm{~min}$ at $72{ }^{\circ} \mathrm{C}$ was performed for final elongation. PCR reaction products were loaded onto $1.5 \%$ agarose gel containing ethidium bromide. ${ }^{33,35}$

Specimens positive for IS6110 were considered as Mycobacterium tuberculosis complex. Samples with negative PCR result based on IS6110 were analyzed for non-tuberculous mycobacteria using PCR-RFLP method.

This gene was amplified using a Nested-PCR with primer pair Tb15: 5'-CGT AYG ACG AAG AGG CCC GT 3' and Tb17: 5'-WAS GGR TCC TCS AGG ACS GC -3'(33).

PCR mixture with a final volume of $50 \mu$ containing 4 Pico moles of Tb15 and Tb17 primer.1.5 mM magnesium chloride, One unit of Taq polymerase (Synagen Corporation), $0.2 \mathrm{mM}$ dNTP, $1.5 \mathrm{mM} 10 \mathrm{X}$ buffer and $1 \%$ DMSO were used. The PCR reaction in Thermal cycler consisted of 30 cycles with annealing temperature of $60{ }^{\circ} \mathrm{C}$.

The second step was performed using primer pairs Tb11: 5'ACCAA CGATGGTGTGTCCA $3^{\prime}$ and Tb12: 5'CTTGTCGAACCGCATACCCT. ${ }^{36}$

PCR reaction with $50 \mu \mathrm{l}$ volume containing 8 Pico moles of Tb11 and Tb12 primer, $1.5 \mathrm{mM}$ magnesium chloride, 1 unit of Taq polymerase enzyme (Synagen Co.), $0.2 \mathrm{mM}$ dNTP, $1.5 \mathrm{mM} 10 \mathrm{X}$ buffer and $2 \%$ DMSO was used.

The conditions of the reaction temperature as follows: the first cycle of $95{ }^{\circ} \mathrm{C}$ for 5 min denaturation primary by 30 cycles of $94^{\circ} \mathrm{C} 30 \mathrm{~s}, 56^{\circ} \mathrm{C}$ $1 \mathrm{~min}$ and $72{ }^{\circ} \mathrm{C}$ in $40 \mathrm{~s}$ and the last cycle $72{ }^{\circ} \mathrm{C}$ for $10 \mathrm{~min}$.

Finally, amplified fragments obtained by PCR on $5.1 \%$ agarose gel were electrophoresed and $439 \mathrm{bp}$ band was absorbed. To analyze the data, SPSS statistical software was used and the frequency and percentages were calculated. MAS-PCR was used to identify mutations in the katG codon 315 and rpoB gene codons 526, 516, 513 and inhA15. ${ }^{37,38}$ In this method, at each allele-specific primer, the $3^{\prime}$ end is positioned for pairing with the associated codons, where most point mutations are found to compare with the wild-type sequence ip37Rv. For each MAS-PCR reaction, a standard $35 \mu \mathrm{l}$ reaction was used.

\section{Results and discussion}

In order to monitor and develop appropriate treatment strategies for mycobacterial infections, the rates of mycobacterial infections (MTBC and NTM clinical isolation) and their drug resistance were investigated during 2011-2018. To investigate drug resistance, resistance profiles of clinical isolates of MTBC and NTMs to two first-line antibiotics, namely INH (isoniazid) and RIF (rifampin) were evaluated during the study. 
Table 2

The number of clinical samples prepared by type of study during the study period 2011-2018.

\begin{tabular}{|c|c|c|c|c|c|c|c|c|c|}
\hline \multirow[t]{2}{*}{ Year Specimen type } & 2011 & \multirow[t]{2}{*}{2012} & \multirow[t]{2}{*}{2013} & \multirow[t]{2}{*}{2014} & \multirow[t]{2}{*}{2015} & \multirow[t]{2}{*}{2016} & \multirow[t]{2}{*}{2017} & \multirow[t]{2}{*}{2018} & \multirow[t]{2}{*}{ total } \\
\hline & МТВ & & & & & & & & \\
\hline CSF & $16(0.52)$ & - & $90(7.96)$ & $93(3.27)$ & $1(0.09)$ & $4(0.21)$ & $3(0.11)$ & $5(0.19)$ & 212(1.34) \\
\hline Sputum & $3013(97.45)$ & $362(90.73)$ & $893(78.96)$ & $660(23.21)$ & $1032(92.14)$ & $1809(93.88)$ & $2571(94.66)$ & $2490(95.73)$ & $12830(81.05)$ \\
\hline BAL & $3(0.1)$ & - & $2(0.18)$ & $52(1.83)$ & $2(0.18)$ & $10(0.52)$ & $7(0.26)$ & $9(0.35)$ & $85(0.54)$ \\
\hline Pleural & $4(0.13)$ & $4(1)$ & $6(0.53)$ & $120(4.22)$ & $1(0.09)$ & $2(0.1)$ & $10(0.37)$ & $5(0.19)$ & $152(0.96)$ \\
\hline bronchia & $4(0.13)$ & $4(1)$ & $5(0.44)$ & $1062(37.35)$ & $2(0.18)$ & $3(0.16)$ & $5(0.18)$ & $2(0.08)$ & $1087(6.87)$ \\
\hline Urine & $4(0.13)$ & - & $3(0.27)$ & $52(1.83)$ & $2(0.18)$ & $5(0.26)$ & $5(0.18)$ & $6(0.23)$ & $77(0.49)$ \\
\hline $\mathrm{g} / \mathrm{w}$ & $7(0.23)$ & $1(0.25)$ & $6(0.53)$ & $381(13.4)$ & $5(0.45)$ & $2(0.1)$ & $4(0.15)$ & $18(0.69)$ & $424(2.68)$ \\
\hline Biopsy & $3(0.1)$ & $1(0.25)$ & $8(0.71)$ & $134(4.71)$ & $8(0.71)$ & $4(0.21)$ & $5(0.18)$ & $6(0.23)$ & $169(1.07)$ \\
\hline Ascites & - & $1(0.25)$ & $45(3.98)$ & $19(0.67)$ & - & - & $2(0.07)$ & - & $67(0.42)$ \\
\hline lavage & $1(0.03)$ & - & - & $5(0.18)$ & $1(0.09)$ & $2(0.1)$ & $1(0.04)$ & - & $10(0.06)$ \\
\hline other & $37(1.2)$ & $26(6.52)$ & $73(6.45)$ & $265(9.32)$ & $66(5.89)$ & $86(4.46)$ & $103(3.79)$ & $60(2.31)$ & $716(4.52)$ \\
\hline total & 3092 & 399 & 1131 & 2843 & 1120 & 1927 & 2716 & 2601 & 15829 \\
\hline
\end{tabular}

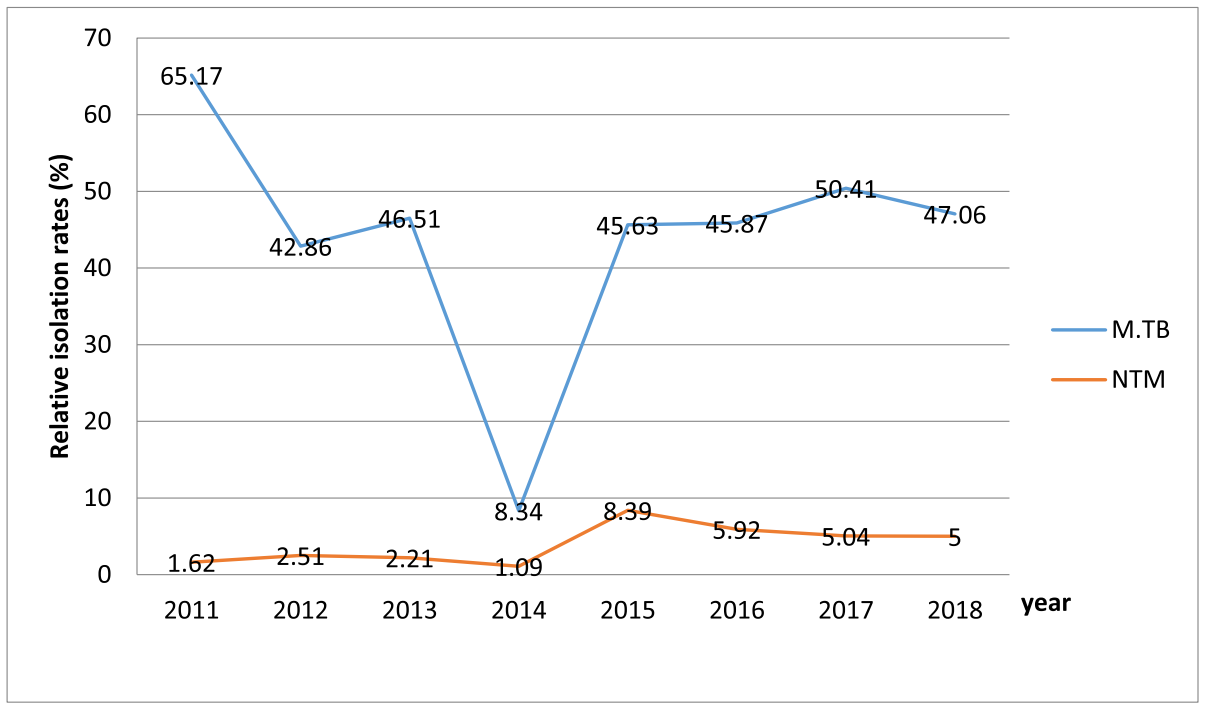

Fig. 1. Reciprocal alteration in the relative isolation rates (\% in y-axis) of M.TB and NTM strains from 2011 to 2018 (X-axis).

Table 3

The number and type of nontuberculous mycobacteria during the study period 2011-2018.

\begin{tabular}{|c|c|c|c|c|c|c|c|c|c|}
\hline Year strain & 2011 & 2012 & 2013 & 2014 & 2015 & 2016 & 2017 & 2018 & Total \\
\hline M.abscessus & - & - & 2 & 1 & 2 & 11 & 14 & 36 & 66 \\
\hline M.atypical & - & - & 1 & - & - & - & - & - & 1 \\
\hline M.avium & - & - & - & 3 & 2 & 2 & 5 & 1 & 13 \\
\hline M.BCG & 1 & - & - & & 3 & - & 9 & 4 & 17 \\
\hline M.bovis.BCG & - & - & - & 11 & - & - & - & - & 11 \\
\hline M.brendly & 1 & - & - & - & - & - & - & - & 1 \\
\hline M.chelonae & 13 & 2 & 2 & 7 & 7 & 4 & 4 & 3 & 42 \\
\hline M.chelonae sub abscessus & - & 2 & - & - & - & - & - & - & 2 \\
\hline M.fortuitum & 3 & - & - & - & - & - & 1 & - & 4 \\
\hline M.genavense & - & - & - & - & - & - & 1 & - & 1 \\
\hline M.gordonae & - & - & - & - & 1 & 2 & - & - & 3 \\
\hline M.intracellular & 1 & - & - & 1 & 12 & 17 & 23 & 14 & 68 \\
\hline M.kansasii & 1 & - & - & 1 & 3 & 7 & 9 & 2 & 23 \\
\hline M.parafortuitum & - & - & - & - & - & - & 1 & - & 1 \\
\hline M.parascrofulaceum & - & - & - & - & - & 2 & - & 2 & 4 \\
\hline M.simiae & 30 & 6 & 20 & 6 & 58 & 69 & 70 & 68 & 327 \\
\hline M.szulgai & - & - & - & 1 & - & - & - & - & 1 \\
\hline Other NTM & - & - & - & - & 6 & - & - & - & 6 \\
\hline Total NTM isolated & 50 & 10 & 25 & 31 & 94 & 114 & 137 & 130 & 591 \\
\hline
\end{tabular}

\section{Positive isolation rate by microscopic smear, mycobacterial culture and PCR method}

During the 8-year study, 15829 samples were collected. Among these samples $(7528 / 15829,47.56 \%)$ mycobacterial isolates including
6937 MTBC (43.82\%) and 591 NTM (3.73\%) were obtained. The number of positive clinical specimens for MTBC and NTM isolates are also listed in Table 1 and Table 2, respectively. MTBC isolation rates were $47.06 \%$ (1224/2601, year 2018) and $65.17 \%$ (2015/3092, year 2011) during the study. It is noteworthy that the NTM positive isolation 
Table 4

Resistance rate of MTB clinical isolates against first-line antituberculosis drug from year 2011-2018.

\begin{tabular}{|c|c|c|c|c|c|c|c|c|}
\hline Year Antibiotic & 2011 & 2012 & 2013 & 2014 & 2015 & 2016 & 2017 & 2018 \\
\hline No. + PCR specimen & 2065 & 181 & 551 & 268 & 605 & 998 & 1506 & 1354 \\
\hline INH (R) & $330(15.98)$ & $53(29.28)$ & $153(27.77)$ & $31(11.57)$ & 221(36.53) & $242(24.25)$ & $333(22.11)$ & 254(18.76) \\
\hline INH (S) & $1740(84.26)$ & 103(56.91) & $373(67.7)$ & $237(88.43)$ & $380(62.81)$ & $753(75.45)$ & $1172(77.82)$ & 1098(81.09) \\
\hline RIF (R) & 257(12.45) & $66(36.46)$ & $151(27.4)$ & $31(11.57)$ & 189(31.24) & 196(19.64) & 239(15.87) & 197(14.55) \\
\hline RIF (S) & $1813(87.8)$ & $120(66.3)$ & $379(68.78)$ & $237(88.43)$ & $412(68.1)$ & $799(80.06)$ & $1266(84.06)$ & $1156(85.38)$ \\
\hline MDR TB & 134(6.49) & $40(22.1)$ & $68(12.34)$ & $4(1.49)$ & $165(27.27)$ & 171(17.13) & $200(13.28)$ & 174(12.85) \\
\hline
\end{tabular}

MDR TB = multidrug resistance tuberculosis, $\mathrm{INH}=$ isoniazid, $\mathrm{RIF}=$ rifampicin.

*No (\%), (R) = resistant $(\mathrm{S})=$ sensitive.

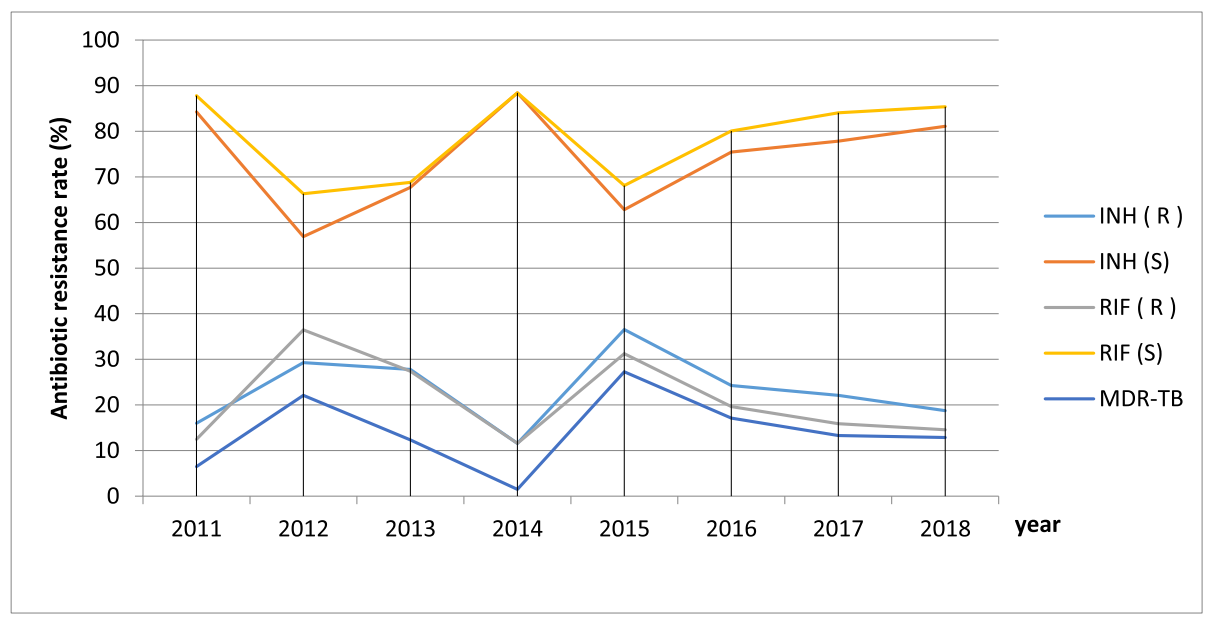

Fig. 2. Resistance rates (\% in y-axis) of 7528 MTB and NTM clinical isolates against the 2 first-line antituberculous drugs, namely INH, RIF during study years (X-axis). M.TB = mycobacterium tuberculosis group, NTM = nontuberculous mycobacteria, MDR TB = multidrug resistance tuberculosis, $\mathrm{INH}=$ isoniazid, $\mathrm{RIF}=$ rifampicin.

rate increased from $1.62 \%(30 / 5092$, year 2011$)$ to $5 \%(266 / 13001$, year 2018).

\section{Distribution of MTB and NTM isolates}

The distribution of MTB and NTM among mycobacterial clinical isolates was evaluated and illustrated in Fig. 1. Among 7528 clinical isolates of Mycobacterium isolates, the frequency of MTBC isolates showed a decreasing trend as ratios decreased from 65.17\% (2015/ $3092)$ in 2011 to $47.06 \%(1224 / 2601)$ in 2018 . In contrast, there is a significant upward trend in NTM isolates, increasing from $1.62 \%$ (50/ $3092)$ in 2011 to $5 \%(130 / 2601)$ in 2018. In particular, the frequency of MTB and NTM isolates during these eight years showed a reciprocal change that approached each other during 2014.

In addition, the number and types of NTM isolates during the years studied are shown in Table 3.

M. simiae with $55.33 \%(327 / 591)$ had the highest number followed by M. intracellular, M. abscessus and $M$. chelonae with $11.51 \%, 11.17 \%$ and $7.11 \%$, respectively.

Our results show that there is a significant interaction change in MTB and NTM isolation rates at the NRITLD center during the study period despite a concomitant decrease in MTB incidence and a parallel increase in NTM incidence. These observations are supported by our previous findings as well as conclusions from other reports, namely that an increase in the isolation of NTM clinical specimens is associated with a decrease in the prevalence of TB. Possible factors leading to this trend toward growth in NTM strains isolation may include increased susceptibility to these strains, increased recognition of NTM infections by physicians and microbiologists, implementation of public health strategies, TB targeting, and it will also enhance the diagnostic methods used in medical laboratories.

Also, the current findings support our earlier suggestion that improving the availability of new techniques that enable accurate identification of NTM strains may lead to further enhancement of NTM detection in the future. ${ }^{39}$ The clinical symptoms of MTB infections often overlap, which is a major problem that needs to be addressed especially when diagnosing NTM infections. ${ }^{40,41}$

In addition, the continued increase in NTM infections makes it difficult to implement a direct TB treatment program, The short-term strategy (DOTS) indicates that tuberculosis only be treated according to the microscopic results of acid fast sputum smear. As a result of the DOTS program, most patients with NTM are likely to be treated with anti-TB drugs when acid fast bacilli are in their sputum smear before their NTM outcome is available. Unfortunately, this can lead to treatment failure or relapse because of differences between bacterial components, drug resistance patterns, clinical manifestations and treatment outcome between NTM and MTB infection despite some similarities. ${ }^{4,14,42,43}$ The aforementioned problems, ranging from initial diagnosis to treatment outcome, are probably all contributing factors to the increase and spread of NTM strains that appear to be reflected in the upregulation pattern identified in the present study. In this context, early and accurate diagnosis of patients with NTM infection is a prerequisite for effective intervention. Standard operating protocols for the diagnosis and identification of NTM susceptibility need to be established in medical laboratories to improve the early detection of NTM infections. This is all the more necessary as the frequency of NTM isolates is constantly and significantly increasing. In addition, potential causes of this increase should be explored to enable public health strategies that can effectively control these emerging NTM infections.

\section{MTBC and NTM clinical isolation antibiotic resistance profiles}

Among the 7528 MTB and NTM isolates, the average resistance to INH ratio was $21 \%$, from $15.98 \%$ in 2011 to $18.76 \%$ in 2018 (Table 4 and Fig. 2). In the case of RIF, the same resistance trend has been increasing and gradually increasing from $12.45 \%$ in 2011 to $14.55 \%$ in 2018. Concerning the average susceptibility of mycobacterial isolates to INH and RIF, it was almost constant and did not show such a change, with $63 \%$ for INH and 78\% for RIF, respectively. Finally, the prevalence of MDR TB during the study period increased steadily from $6.49 \%$ 
(134/2065) in 2011 to $12.58 \%(174 / 1354)$ in 2018

The subject of the continued expansion of MDR TB, which causes intolerable tuberculosis and leads to patient death, is a worldwide study $(21,44)$. About $5 \%$ of global tuberculosis cases have MDR tuberculosis with about $3.5 \%$ incidence of new MDR tuberculosis. ${ }^{14,15,44,45}$ The average prevalence of MDR TB in our study, approximately $14.18 \%$, which is much higher than the global average of $3.5 \%$ of MDR TB, and this is very alarming. The MDR tuberculosis rate has remained stable due to the high level of resistance to INH and RIF. Despite global efforts to reduce the number of TB cases, the spread of MDR TB is still a threat, leading to the spread of TB and a significant number of deaths. MDR TB treatment is expensive and time-consuming for both patients and physicians, and is often associated with a high incidence of mismatches and side effects $(21,44)$. Therefore, more effort and more effective strategies are needed to control TB in different areas. Knowledge and information on the prevalence of mycobacteria, the trend of antibiotic resistance, advanced identification techniques and drug susceptibility testing are important for the effective control of mycobacterial infections. Based on the findings of the present study on mycobacterial infections and drug allergies that have been documented for 8 years, we conclude and recommend that the diagnosis, identification and sensitivity testing for NTM should be standardized and integrated in clinical systems.

\section{Declaration of competing interest}

There are no conflicts of interest

\section{References}

1. Tortoli E. Impact of genotypic studies on mycobacterial taxonomy: the new mycobacteria of the 1990s. Clin Microbiol Rev. 2003;16(2):319-354.

2. Joao I, Cristovao P, Antunes L, Nunes B, Jordao L. Identification of nontuberculous mycobacteria by partial gene sequencing and public databases. International journal of mycobacteriology. 2014;3(2):144-151.

3. Cramer J, Sudeck H, Burchard G. Pulmonary infection with rapidly growing mycobacteria in a singer with achalasia: a case report. J Infect. 2007;54(4):e219-e221.

4. Shiau M-Y, Lee M-S, Huang T-L, Tsai J-N, Chang Y-H. Mycobacterial prevalence and antibiotic resistance frequency trends in Taiwan of mycobacterial clinical isolates from 2002 to 2014. Medicine. 2016;95(12).

5. !!! INVALID CITATION !!! [5-11].

6. Simons S, Van Ingen J, Hsueh P-R, et al. Nontuberculous mycobacteria in respiratory tract infections, eastern Asia. Emerg Infect Dis. 2011;17(3):343.

7. Wang $\mathrm{H}-\mathrm{x}$, Jun $\mathrm{Y}$, Min H, et al. Nontuberculous mycobacteria: susceptibility pattern and prevalence rate in Shanghai from 2005 to 2008. Chin Med J. 2010;123(2):184-187.

8. Martín-Casabona N, Bahrmand A, Bennedsen J, et al. Non-tuberculous mycobacteria: patterns of isolation. A multi-country retrospective survey. Int J Tuberc Lung Dis. 2004;8(10):1186-1193.

9. Gayathri R, Therese KL, Deepa P, Mangai S, Madhavan H. Antibiotic susceptibility pattern of rapidly growing mycobacteria. J Postgrad Med. 2010;56(2):76.

10. Rafi A, Moaddab S, Radmehr R. Drug resistance study of Mycobacterium tuberculosis strains and mycobacteria other than tubercle bacilli strains to ofloxacin and ciprofloxacin isolated from patients admitted to research center for TB and pulmonary diseases of Tabriz. Pharm Sci. 2009;15(3):241-246.

11. Vacher S, Pellegrin J, Leblanc F, Fourche J, Maugein J. Comparative antimycobacterial activities of ofloxacin, ciprofloxacin and grepafloxacin. $J$ Antimicrob Chemother. 1999;44(5):647-652.

12. Olivier KN, Weber DJ, Wallace Jr RJ, et al. Nontuberculous mycobacteria: I: multicenter prevalence study in cystic fibrosis. Am J Respir Crit Care Med. 2003;167(6):828-834

13. Farnia P, Masjedi MR, Mohammadi F, et al. Colorimetric detection of multidrug resistant or extensively drug-resistant tuberculosis by use of malachite green indicator dye. J Clin Microbiol. 2008;46(2):796-799.

14. Mirsaeidi MS, Tabarsi P, Farnia P, et al. Trends of drug resistant Mycobacterium tuberculosis in a tertiary tuberculosis center in Iran. Saudi Med J. 2007;28(4):544

15. Merza MA, Farnia P, Tabarsi P, Khazampour M, Masjedi MR, Velayati AA. Anti-tuberculosis drug resistance and associated risk factors in a tertiary level TB center in Iran: a retrospective analysis. The Journal of Infection in Developing Countries. 2011;5:511-519 07

16. Kwon Y-S, Koh W-J. Distinguishing between pulmonary tuberculosis and non-tuberculous mycobacterial lung disease. Int J Tuberc Lung Dis. 2014;18(6):633.
17. Hashemi-Shahraki A, Bostanabad SZ, Heidarieh P, et al. Species spectrum of nontuberculous mycobacteria isolated from suspected tuberculosis patients, identification by multi locus sequence analysis. Infect Genet Evol. 2013;20:312-324.

18. Tabarsi $\mathrm{P}$, Baghaei $\mathrm{P}$, Farnia $\mathrm{P}$, et al. Nontuberculous mycobacteria among patients who are suspected for multidrug-resistant tuberculosis-need for earlier identification of nontuberculosis mycobacteria. Am J Med Sci. 2009;337(3):182-184.

19. Velayati AA, Rahideh S, Nezhad ZD, Farnia P, Mirsaeidi M. Nontuberculous mycobacteria in Middle East: current situation and future challenges. International Journal of Mycobacteriology. 2015;4(1):7-17.

20. Baghaei P, Tabarsi P, Farnia P, et al. Pulmonary disease caused by Mycobacterium simiae in Iran's national referral center for tuberculosis. The Journal of Infection in Developing Countries. 2012;6:23-28 01.

21. Mirsaeidi M, Farnia P, Sadikot R, Hsueh P-R, Aliberti S. Nontuberculous mycobacteria: epidemiologic, mycobacteriologic, and clinical aspects. BioMed Res Int. $2015 ; 2015$.

22. Prevots DR, Shaw PA, Strickland D, et al. Nontuberculous mycobacterial lung disease prevalence at four integrated health care delivery systems. Am J Respir Crit Care Med. 2010;182(7):970-976.

23. Van Ingen J, Ferro BE, Hoefsloot W, Boeree MJ, Van Soolingen D. Drug treatment of pulmonary nontuberculous mycobacterial disease in HIV-negative patients: the evidence. Expert Rev Anti-infect Ther. 2013;11(10):1065-1077.

24. Griffith DE, Aksamit T, Brown-Elliott BA, et al. An official ATS/IDSA statement: di agnosis, treatment, and prevention of nontuberculous mycobacterial diseases. Am $J$ Respir Crit Care Med. 2007;175(4):367-416.

25. Keating M, Daly J, Practice AIDCo. Nontuberculous mycobacterial infections in solid organ transplantation. Am J Transplant. 2013;13(s4):77-82

26. Knoll B, Kappagoda S, Gill R, et al. Non-tuberculous mycobacterial infection among lung transplant recipients: a 15-year cohort study. Transpl Infect Dis. 2012;14(5):452-460

27. Arend SM, van Soolingen D, Ottenhoff TH. Diagnosis and treatment of lung infection with nontuberculous mycobacteria. Curr Opin Pulm Med. 2009;15(3):201-208.

28. Addo KK, Addo SO, Mensah GI, Mosi L, Bonsu FA. Genotyping and drug susceptibility testing of mycobacterial isolates from population-based tuberculosis prevalence survey in Ghana. BMC Infect Dis. 2017;17(1):743.

29. Velayati AA, Farnia P, Mozafari M, Mirsaeidi M. Nontuberculous mycobacteria isolation from clinical and environmental samples in Iran: twenty years of surveillance. BioMed Res Int. 2015;2015.

30. Lai C-C, Tan C-K, Chou C-H, et al. Increasing incidence of nontuberculous mycobacteria, Taiwan, 2000-2008. Emerg Infect Dis. 2010;16(2):294.

31. Chien J-Y, Lai C-C, Sheng W-H, Yu C-J, Hsueh P-R. Pulmonary infection and colonization with nontuberculous mycobacteria, Taiwan, 2000-2012. Emerg Infect Dis. 2014;20(8):1382.

32. da Costa ARF, Lopes ML, Furlaneto IP, de Sousa MS, Lima KVB. Molecular identification of nontuberculous mycobacteria isolates in a Brazilian mycobacteria reference laboratory. Diagn Microbiol Infect Dis. 2010;68(4):390-394.

33. Derakhshani Nezhad Z, Farnia P, Sheikholslami FM, et al. Prevalence of non-tuberculosis mycobacteria in patients referring to mycobacteriology research center of Iran. Scientific Journal of Kurdistan University of Medical Sciences. 2014;19(2):31-39.

34. Velayati AA, Farnia P, Mozafari M, et al. Molecular epidemiology of nontuberculous mycobacteria isolates from clinical and environmental sources of a metropolitan city. PLoS One. 2014:9(12):e114428.

35. Telenti A, Marchesi F, Balz M, Bally F, Böttger E, Bodmer T. Rapid identification of mycobacteria to the species level by polymerase chain reaction and restriction enzyme analysis. J Clin Microbiol. 1993;31(2):175-178.

36. Farnia P, Masjedi M, Nasiri B, et al. Instability of IS6110 patterns in multidrug-resistant strains of Mycobacterium tuberculosis. Epidemiol Infect. 2007; 135(2):346-352.

37. Hwang H-Y, Chang C-Y, Chang L-L, Chang S-F, Chang Y-H, Chen Y-J. Characterization of rifampicin-resistant Mycobacterium tuberculosis in taiwan. $J$ Med Microbiol. 2003;52(3):239-245

38. Baker L, Brown T, Maxwell O, et al. Molecular analysis of isoniazid-resistant Mycobacterium tuberculosis isolates from England and Wales reveals the phylogenetic significance of the ahpC - 46A polymorphism. Antimicrob Agents Chemother. 2005;49(4):1455-1464

39. Tsai C-F, Shiau M-Y, Chang Y-H, et al. Trends of mycobacterial clinical isolates in Taiwan. Trans R Soc Trop Med Hyg. 2011;105(3):148-152

40. Gopinath K, Singh S. Non-tuberculous mycobacteria in TB-endemic countries: are we neglecting the danger? PLoS Neglected Trop Dis. 2010;4(4):e615.

41. Nasiri MJ, Dabiri H, Darban-Sarokhalil D, Rezadehbashi M, Zamani S. Prevalence of drug-resistant tuberculosis in Iran: systematic review and meta-analysis. Am J Infect Contr. 2014;42(11):1212-1218

42. Wallace Jr RJ, Brown BA, Griffith DE. Nosocomial outbreaks/pseudo outbreaks caused by nontuberculous mycobacteria. Annu Rev Microbiol. 1998;52(1):453-490.

43. Ellis M. Mycobacteria other than Mycobacterium tuberculosis. Curr Opin Infect Dis. 1988;1(2):252-271.

44. Baghaei P, Tabarsi P, Dorriz D, et al. Adverse effects of multidrug-resistant tuberculosis treatment with a standardized regimen: a report from Iran. Am J Therapeut. 2011;18(2):e29-e34

45. Organization WH. Global Tuberculosis Report 2013. World Health Organization; 2014. 